\title{
Securing Body Sensor Networks: Sensor Association and Key Management
}

\author{
Sye Loong Keoh \\ Dept. of Info. \& System Security, Philips Research \\ High Tech Campus 34, 5656 AE, Eindhoven \\ Email: sye.loong.keoh@philips.com
}

\author{
Emil Lupu and Morris Sloman \\ Dept. of Computing, Imperial College London \\ London SW7 2RH \\ Email: \{e.c.lupu, m.sloman\}@imperial.ac.uk
}

\begin{abstract}
Body Sensor Networks can be used to continuously monitor patients' health. However, secure association of sensors with the patient and key management for providing integrity and confidentiality to the sensor readings are essential. We propose a secure discovery protocol based on the synchronised LED blinking pattern, to enable healthcare workers to authorise the sensor-to-patient association. We also propose a novel key distribution and management scheme that uses keychains to establish group keys for body sensor networks and caters for group key update and re-keying to adapt to membership changes. These protocols have been implemented to demonstrate their feasibility and an initial performance evaluation is presented.
\end{abstract}

\section{INTRODUCTION}

Wireless Body Sensor Networks (BSNs) with portable devices such as smart phones enable continuous efficient monitoring and management of post-operative and chronicallyill patients to enable early release from hospital. Healthcare personnel can then be automatically alerted if the patient's condition deteriorates. BSNs must ensure confidentiality, integrity and availability of the physiological data, as wireless sensor networks are susceptible to passive eavesdropping, packet injection, and vulnerable to many other security attacks. Furthermore, the design of the security protocols must carefully balance satisfying the security requirements with limiting the power and computational requirements. To securely establish BSNs we must first ensure that only designated sensors are associated with the patient and only by an authorised party. For example, a nurse is permitted to attach an approved ECG sensor to monitor the heart-rate of a post-operative patient, while arbitrary nearby ECG sensors on other patients must not be associated.

The second security goal is to preserve the confidentiality and integrity of the medical data, which is susceptible to eavesdropping when transmitted wirelessly. Sensor readings must therefore be encrypted and given the sensors' limited capabilities, the use of a shared group key is desirable. A lightweight key management scheme is needed to use less computationally intensive cryptographic operations and reduce power consumption by minimising messages exchanges.

Thirdly, managing group membership is required to facilitate addition and removal of sensors from the BSN, and

This research was supported by UK EPSRC BiosensorNet (Grant EP/C547586/1) and Aedus2 (Grant EP/E025188/1) renewing the group key when necessary. Existing sensors may fail permanently or temporarily become disconnected from the BSN whereas new sensors may be added according to medical needs. There is a need to distinguish between temporary and permanent disconnection, to establish persistent pairings that survive transient disconnection and to renew the group key when a sensor is permanently detached. We propose a novel secure sensor discovery protocol that integrates the BLIG [1] sensor association scheme, with an efficient key distribution and key management scheme using keychains to establish a shared secret group key.

The paper is organised as follows: Section II discusses related work and its limitations. Section III discusses the threat model. Section IV, describes sensor discovery and association, while Section V describes the key distribution and management. We present the prototype implementation and results in Section VI and discuss trade-offs in Section VII. Conclusions and future work are presented in Section VIII.

\section{RELATED WORK}

BLIG [1] uses synchronised blinking of LEDs to enable a healthcare worker to visually verify the correct sensors grouping on a patient. It uses short range communication (i.e., $<0.5 \mathrm{~m}$ ) to discover new sensors, establish the patient's identity and map between the patient's true id and the sensor group. We have enhanced BLIG for more robust sensor discovery, association, sensor authentication and key management.

Pre-shared symmetric key protocols are used in large scale sensor networks [2], [3]. A key-share is loaded in each node and used to derive a common secret key. In the hospital, this is insufficient as groups overlap in their wireless range and only the correct sensors must be associated with the patient. These protocols also require intensive computation to replace the group key when membership changes.

Balfanz [4] uses a secure-limited channel, e.g., infrared to exchange public-keys between parties in a pre-authentication phase before authentication. However, this requires dedicated hardware and/or hosting the pre-authentication phase in a confined area, which would be difficult in a hospital ward. Human confirmation of correct association is also difficult when based on public-keys and without visual cues.

The resurrecting duckling protocol [5] establishes a masterslave relationship between devices where the first device in 
contact with a sensor becomes its master and can upload policies to the sensor that permit interactions with other devices. Sensors from previous patients have to be explicitly dis-associated by the master before reuse by other patients which may not always be practical in hospitals.

Jiang [6] uses self-certified keys (SCK) and Elliptic Curve Cryptography (ECC) to establish pair-wise keys for authentication. Each sensor establishes a secret with the user based on the secret information pre-loaded by a key distribution centre (KDC). To achieve sensor to patient association, each patient's BSN would require different ECC curve parameters (as each $\mathrm{BSN}$ is a domain) which would be impractical for hundreds of BSNs in a hospital.

Zhu [7] introduced a privacy-preserving discovery service by exchanging code words between the service provider and clients. However, a shared secret must be established a priori.

\section{THREAT MODEL}

It is not easy to confine short range wireless interactions in hospital wards. Data confidentiality may be compromised through eavesdropping. Data integrity may be compromised by injecting data into the wireless channel or simply interfering with the wireless transmission. Sensors may be associated with the wrong patient either mistakenly or maliciously (e.g., through impersonation). Medical data is confidential and would interest many attackers including employers, insurance companies, personal enemies and unscrupulous media for newsworthy persons.

Passive Eavesdropping. Wireless channels are susceptible to passive eavesdropping and message interception. Whilst traffic can be encrypted, sensor data is vulnerable to cryptanalysis as measurements fall within known ranges, and chosen ciphertext attacks become possible. Therefore, the encryption key must be regularly renewed and used for short time periods.

Active Attacks. Active attacks include injecting messages into the wireless channel, replaying or altering messages. Spurious messages injected into the network, e.g., sending false sensor readings will disrupt the accuracy of the measurements. Message replays or modification of messages, to cause a medication overdose, pose a serious risk to the patient. Thus, authentication, authorisation and message freshness are necessary to ensure the confidentiality and integrity of the transmitted data.

Impersonation and Masquerading. Impersonation would typically occur when an attacker in the guise of a sensor joins or claims to be part of a patient's BSN to eavesdrop on data and/or report false data. Authentication is essential to prove the sensor's membership in the BSN.

Denial of Service Attack. We do not attempt to address physical Denial-of-Service (DoS) attacks by frequency jamming [8], but are solely concerned with detecting malicious sensors that repeatedly attempt to join a BSN, thus aiming to deplete resources and prevent genuine sensors from joining.

\section{Secure Sensor Association}

Post-operative patients typically need continuous monitoring whilst in hospital or at home e.g. ECG sensors monitor heart condition, $\mathrm{SpO}_{2}$ sensors monitor oxygen saturation, and other sensors observe blood pressure and body temperature. A device such as PDA or mobile phone is often used as a controller to coordinate communication and manage the sensors in the BSN. When attaching a new sensor, the healthcare worker switches on the sensor, enabling discovery by the controller. However, nearby sensors will also be discovered and solely based on sensor type and credentials, the controller cannot decide if the sensor should be associated with its patient. An explicit action is thus required to identify the correct association. Instead of using short-range confined communication or physical contact to distinguish the sensor, we use the BLIG approach [1] where sensor and controller synchronise their LED blinking in an agreed pattern. This provides an authorised healthcare worker with the visual representation of the association, that she can then explicitly authorise. The discovery protocol also establishes a secret key between each sensor admitted to the BSN and the patient's controller, it is then used to distribute the group key. The next section presents our assumptions and introduces the cryptographic notation whilst the protocol is described in Section IV-B.

\section{A. Assumptions and Notation}

We consider the hospital as the root Certification Authority (CA) issuing attribute certificates to its staff, patients, devices and sensors certifying their roles and attributes. Appropriate certificates and private keys are pre-loaded into devices and sensors before release for use.

Sensors attached to the patient are discovered sequentially, by the patient's controller and a complete iteration of the protocol is necessary for each sensor. Otherwise, an attacker sensor could "lie" and copy the neighbour's blinking at the same time as the neighbour is being associated.

Medical sensors are trusted to generate good pseudo random numbers using accelerometers or input from other noisy sensors to determine a seed for the generator.

Authenticated healthcare workers and patients i.e. who present valid attribute certificates issued by the hospital are assumed well-behaved. In particular, healthcare workers are trusted to correctly authorise sensor-to-patient associations. The protocols do not attempt to deal with malicious insiders.

Furthermore, sensors that have become detached from the $\mathrm{BSN}$, are trusted to detect this and wipe state information such as encryption keys and received messages from their RAM. The notation used in our protocol is given in Table I.

\section{B. Secure Discovery Protocol}

Figure 1 summarises the secure discovery protocol using a sequence diagram. Broadly the protocol is divided into three phases: mutual authentication, information exchange and sensor association.

1) Mutual Authentication Phase: The patient's controller and the healthcare worker's device mutually authenticate each other to establish a session context in which the healthcare worker becomes the sole entity permitted to associate sensors with the patient. The patient's controller periodically 


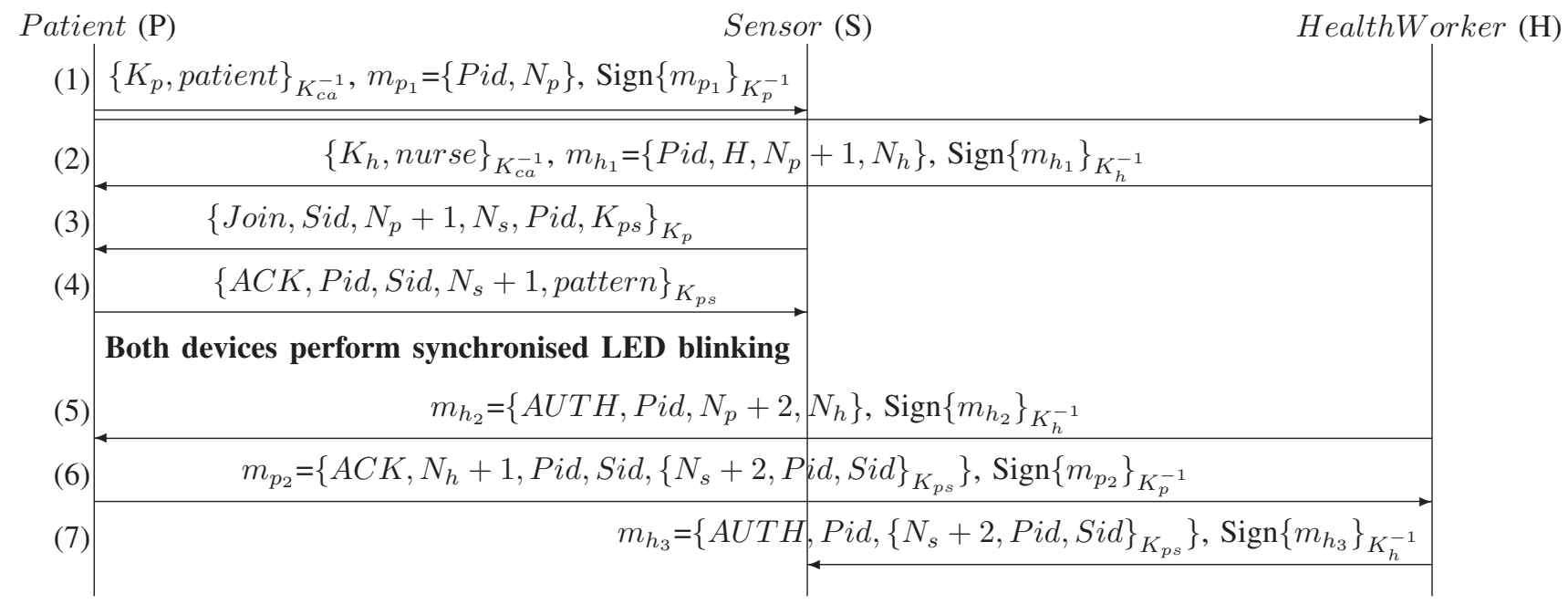

Fig. 1. The secure discovery protocol.

TABLE I

NOTATION USED

\begin{tabular}{l|l}
$H$ & Healthcare worker's device \\
$P$ & patient's controller \\
$S$ & Wireless medical sensor \\
$C A$ & Root Certification Authority Hospital \\
$K_{x}, K_{x}^{-1}$ & Public and private key of $x$ \\
$\left\{K_{x}, a t t\right\}_{K_{y}^{-1}}$ & Attribute certificate bound to $K_{x}$, signed by $K_{y}^{-1}$ \\
$\{m\}_{K_{x}}$ & Encryption of $m$ using public-key of $x$ \\
$\{m\}_{K_{a b}}$ & Encryption of $m$ using secret key $K_{a b}$ \\
$\left.\operatorname{Sign}_{a b}\right\}_{K_{x}^{-1}}$ & Signing hash of $m$ using private-key of $x$ \\
$N_{x}$ & Nonce of $x$ \\
$P i d$ & Unique identifier of BSN \\
Sid & Unique identifier of Sensor
\end{tabular}

broadcasts message (Msg l) to discover sensors and devices nearby. Msg 1 consists of a certificate binding the attribute 'patient' to the patient's public-key, $\left\{K_{p}, \text { patient }\right\}_{K^{-1}}$ as well as a signed message $m_{p_{1}}$ containing the BSN id, Pid and a nonce $N_{p}$. This enables the healthcare worker to verify the signature and the attribute (role) of the patient's controller. The healthcare worker then authenticates itself to the patient's controller by sending Msg 2 which consists of its attribute certificate, $\left\{K_{h}, \text { nurse }\right\}_{K_{c a}^{-1}}$ and a signed message $m_{h_{1}}$. In $m_{h_{1}}$, nonce $N_{p}+1$ guarantees message freshness and $N_{h}+1$ is used to detect replay of $M s g$ 2. Note that the healthcare worker could also authenticate itself to the sensor, but this is not necessary as no interaction between them is needed at this stage.

2) Information Exchange Phase: The sensor and the patient's controller establish a shared secret-key with each other. The LED blinking pattern is sent to the sensor encrypted with a symmetric key. Msg 1 enables the sensor to verify the patient's attribute certificate and public-key, $K_{p}$. The sensor then sends a join request (Msg 3) encrypted with the patient's public-key to the patient. Msg 3 contains $N_{s}$ that guarantees freshness of the message, and Sid as the sensor's ID. The sensor also generates a secret key $K_{p s}$. The patient's controller decrypts the received join request using the private key and retrieves the secret-key. Instead of using public-key encryption, the patient uses the secret-key, $K_{p s}$ to encrypt a blinking pattern and sends it to the sensor in Msg 4. We rely on the sensor to generate a secret key $K_{p s}$ instead of the patient's controller to reduce message exchanges. Furthermore, only the sensor needs to authenticate the patient, but not vice versa as we rely on the healthcare worker to pair the correct devices together. If the patient's controller were to generate $K_{p s}$, the sensor must convey its public-key to the patient so that the patient's controller can securely transmit $K_{p s}$ to the sensor. This would require four messages instead of the original three; the number of cryptographic operations also increases as the sensor needs to generate and verify signatures in $M s g$ 1, 2, 3 as well as decrypting $M s g 3$ using public-key cryptography before the secret key, $K_{p s}$ is first used.

3) Sensor Association Phase: Only the patient's controller and the sensor know the chosen blinking pattern to display for a specified period of time. After observing the synchonised blinking, the healthcare worker authorises the association by sending an $A U T H$ message (Msg 5) to the patient's controller, which then acknowledges it in $M s g$ 6. $N_{p}+2$ and $N_{h}$ in $M s g$ 5 guarantee the freshness of the authorisation message. The healthcare worker's device needs the sensorID for the $A U T H$ message, hence the $A C K$ message (Msg 6) from the patient contains $\mathrm{Sid}$, as well as a commitment encrypted with $K_{p s}$ that only the sensor can decrypt. $N_{s}+2$ in the commitment is used to prevent replay. Msg 7 is an $A U T H$ message sent by the healthcare worker to the sensor confirming its association with the patient. The sensor can decrypt the commitment and verify freshness of $M s g 7$ by checking $N_{s}+2$. Finally, $K_{p s}$ is used as the shared secret-key between the sensor and the patient's controller. The BAN logic proof that both the sensor and the patient's controller believe the secret-key they have derived at the end of the protocol is described in [9].

\section{Key Management}

Communication between the sensor and the patient's controller can be encrypted using the shared secret key, $K_{p s}$. 
However, no keys are shared between sensor pairs and routing all communication via the patient's controller would be inefficient. Moreover, medical events and network changes would typically be conveyed to all devices in the BSN network. Pointto-point notifications incur redundancy and overhead as the same message must be encrypted $n$ times and then sent to $n$ parties. Encrypted broadcast provides an effective scheme for constrained environments that mostly consist of sensors with scarce resources. A shared group key, $G$ can be established to enable all parties in the BSN to communicate with each other directly. This is based on the assumption that only authenticated, hospital approved sensors have been included in the BSN, so they are well behaved and do not impersonate other sensors in the same BSN. The key distribution and management scheme we propose does not rely on public-key cryptography to distribute initial keying materials, but uses symmetric-key cryptography and computation of hashes to significantly reduce computational requirements on sensors.

Two one-way hash chains are used to generate shared group keys. Two keys, one from each hash chain, are concatenated together and the result is hashed to produce the group key. This key can be renewed by advancing both keychains forward to obtain the previous key from the corresponding hash chain. The next group key is then similarly generated by hashing the concatenated keys from the chains.

\section{A. Establishing a Group Key, $G_{i}$}

The patient's controller in the BSN is responsible for the key distribution and management as it typically has higher computational capability and already shares secret keys with each sensor. It generates two key chains [10] each consisting of $n$ keys using a one-way hash function, e.g., SHA-1. A random number is generated as the initial key and the hash function is applied to the initial key to generate the next key. The next key is then hashed repeatedly for $n-1$ times to produce the keychain. The first $k$ chain contains keys $K_{1}, K_{2}, \ldots, K_{n}$ and the second $x$ chain contains $X_{1}, X_{2}, \ldots, X_{n}$.

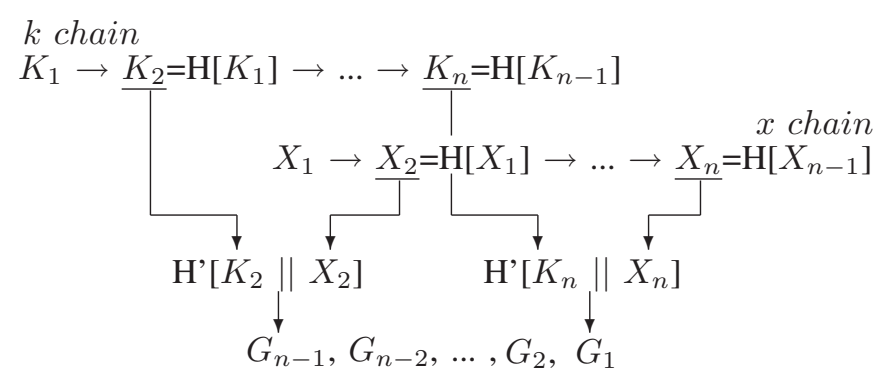

Fig. 2. Generating group keys.

As shown in Figure 2, both keychains are used in reverse order where $K_{n}$ and $X_{n}$ are disseminated, encrypted using $K_{p s}$, the secret-key shared between the patient's controller and the sensor. On receipt, each sensor computes the group secret key, $G_{i}$ by taking the hash of the concatenation of $K_{n}$ and $X_{n}$. This key may be changed whenever a new sensor or device is added, but it must be renewed when an existing sensor is removed from the BSN or when the group key has been used for an extended period of time (c.f. Section V-B). Our key management scheme provides forward secrecy in that compromising any of the $G_{i}$ keys does not reveal sufficient information to compute previous/future group keys.

\section{B. Re-Keying or Key Update}

When a new sensor is discovered, the patient's controller conveys the current $K_{i}$ and $X_{i}$, encrypted with the shared secret-key, $K_{p s}$ to enable the new sensor to compute the current group key, $G_{i}$.

When a sensor leaves the BSN, the group key must be renewed. The patient's controller advances the keychains to obtain $K_{i-1}$ and $X_{i-1}$ from their respective chain. As shown in Figure 3, $X_{i-1}$ is encrypted with the current $X_{i}$, while $K_{i-1}$ is encrypted with $K_{i}$, then broadcast to all sensors/devices in a key update message. Upon receipt, the sensors compute $\mathrm{H}\left[K_{i-1}\right]$ and $\mathrm{H}\left[X_{i-1}\right]$ and ensure that the hash values match the current $K_{i}$ and $X_{i}$ respectively. This authenticates the source and contents of the key update message as only the patient's controller knows the keychains. The sensors then derive the new group key, $G_{i-1}$ using H' $\left[\left(K_{i-1} \| X_{i-1}\right)\right]$.

$$
\begin{array}{lc}
\ldots, \frac{K_{i-1}}{\llcorner}, K_{i}, \ldots & \text { Sensors compute } \\
\left\{X_{i-1}\right\}_{X_{i}},\left\{K_{i-1}\right\}_{K_{i}} \longrightarrow & \mathrm{H}\left[\left(K_{i-1} \| X_{i-1}\right)\right] \\
\ldots, & \text { as the new group key } \\
\ldots &
\end{array}
$$

Fig. 3. Renewing the group key.

If a sensor is detected to have been compromised, a new group key must be derived. The patient's controller broadcasts the following:

$\left\{s_{1},\left\{K_{i-1}, X_{i-1}, N_{p}\right\}_{K_{p s_{1}}}\right\},\left\{s_{2},\left\{K_{i-1}, X_{i-1}, N_{p}\right\}_{K_{p s_{2}}}\right\}, \ldots$, $\left\{s_{n},\left\{K_{i-1}, X_{i-1}, N_{p}\right\}_{K_{p s_{n}}}\right\}$

The compromised sensor's key is not used, so it will not be able to obtain $K_{i-1}$ and $X_{i-1}$, but other sensors can use their respective secret-key to decrypt the message and compute the new group key. $N_{p}$ is used to detect replay of the message. Detecting that a node has been compromised, is very difficult, usually based on anomalous behavior and is not covered here.

When all keys in the keychains have been used up, the patient's controller generates two new keychains and similarly conveys the first key of both keychains $\left(K_{n}^{\prime}\right.$ and $\left.X_{n}^{\prime}\right)$ to all sensors encrypted with each individual sensor's secret-key to ensure that $K_{n}^{\prime}$ and $X_{n}^{\prime}$ are from the patient's controller as encrypting them with the group key does not prove source authenticity. Unlike TESLA [11] which uses keychains for authenticated broadcast, we use keychains to generate group keys for encryption. This has the advantage of efficient and effective authentication of the source of key update messages when re-keying or distributing key updates without relying on public-key cryptography.

\section{Managing Sensor's Intermittent Connectivity}

Transient failures occur if a sensor temporarily moves out of communication range and leaves the BSN for a short 
period. When rejoining the BSN they must still be able to communicate with other devices in the BSN so they have to obtain the latest $K_{i}$ and $X_{i}$ if they have missed the key update messages. We have devised a simple protocol to rejoin a BSN by requiring the patient's controller to maintain the secretkey with the sensor for an extended period of time after the sensor has left. For example, the group key could have been renewed multiple times if the BSN's membership changes after the sensor has left, so the sensor will not have received any key updates from the patient's controller to renew its group key. When it rejoins, it proves membership using its secret-key, $K_{p s}$. It sends a challenge containing its $S i d$ and a nonce $N_{s}$ encrypted with $K_{p s}$ to the patient's device, which responds by encrypting the current $K_{i}$ and $X_{i}$ together with $N_{s}+1$ using $K_{p s}$. The sensor can authenticate the message as it only shares the secret-key with the patient's controller. This scheme avoids the high computation overheads of the full discovery protocol.

\section{IMPLEMENTATION AND EVALUATION}

\section{A. Elliptic Curve Cryptography Parameters}

Both the secure discovery and key management protocols have been implemented on Tmote Sky ${ }^{1}$ using the Elliptic Curve Cryptography (ECC) library, TinyECC version 0.3 [12]. We used the recommended 160-bit Elliptic Curve domain parameters over $F_{p}$ associated with verifiably random parameters, i.e., secpl60rl. A wide range of parameters can be selected from [13] and a base point $G$ is chosen.

\section{B. ElGamal Implementation}

The public key encryption used to encrypt Msg 3 in the secure discovery protocol is implemented using the ElGamal scheme [14]. The plaintext message is first embedded onto the elliptic curve $E$ as a point, $P_{m}$. The sender then chooses a random bit pattern, $r$ and computes two points, $P_{r}=r G$ where $G$ is the base point, and $P_{h}=P_{m}+r P_{B}$ where $P_{B}$ is the public key of the receiver.

The sensor sends both points, $P_{r}$ and $P_{h}$ to the patient device, which extracts the message point by computing $P_{s}$ $=k_{B} P_{r}$ where $k_{B}$ is the private key of the receiver. It then substracts this from $P_{h}$ to get $P_{m}=P_{h}-P_{s}$. By expanding this equation, we show that $P_{m}$ can be recovered as follows:

$$
P_{m}=P_{h}-P_{s}
$$$$
\text { since } P_{h}=P_{m}+r\left(k_{B} G\right) \text { where } r P_{B}=r\left(k_{B} G\right)
$$

we derive that $P_{m}=P_{m}+r\left(k_{B} G\right)-k_{B}(r G)$

Based on the BigInteger library in TinyECC, we have implemented ElGamal encryption for TinyOS version 1.0x.

\section{Skipjack Symmetric Key Encryption}

We initially considered using Tmote Sky's on-chip Advance Encryption Standard (AES) library for symmetric-key encryption of data transfers. However, it only performs standalone encryption and does not support stand-alone decryption, presumably as the manufacturer assumed that all the data from the motes will be sent to a sink node. Although it supports

\footnotetext{
${ }^{1}$ Runs TinyOS and has 16-bit, $8 \mathrm{MHz}$ Texas Instruments MSP430 processor with $48 \mathrm{~KB}$ of ROM and $10 \mathrm{~KB}$ of RAM
}

inline AES encryption at the MAC layer, this is not suitable as we require application layer encryption to be able to select a specific key based on the destination. The software AES implementation on the mote also has unsatisfactory performance [15]. Consequently, we modified the MicaZ specific Skipjack algorithm implemented in TinySec [15] for Tmote Sky. All messages are encrypted using the Skipjack algorithm with a symmetric key. The hash function SHA-1 produces 160-bit output which fits nicely into the key size of Skipjack. We used the Skipjack Cipher Block Chaining (CBC) mode with a block size of 8 bytes and non-repeating Initialisation Vector (IV). The accelerometer reading of the mote has been used as the seed to generate the initial IV to produce different ciphertext from the same plaintext.

\section{Measurements and Evaluation}

TABLE II

LATENCY FOR VARIOUS SECURITY OPERATIONS ON TMOTE SKY

\begin{tabular}{l|c} 
Security Operations & Time \\
\hline Public-key Encryption & $9.53 \mathrm{~s}$ \\
Public-key Decryption & $5.28 \mathrm{~s}$ \\
Signature Generation & $4.26 \mathrm{~s}$ \\
Signature Verification & $5.45 \mathrm{~s}$ \\
\hline Skipjack Encryption & $150 \mu \mathrm{s}$ \\
Skipjack Decryption & $90 \mu \mathrm{s}$
\end{tabular}

Table II shows the execution time of various security operations on Tmote Sky. ElGamal takes 9.53s (variance is 0.167 ) to encrypt a 52 bytes message, while decryption takes 5.28s (variance is 0.00004), as the sender needs to compute two points, $P_{r}=r G$ and $r P_{B}$. Note that, according to [12], digital signature generation and verification take $4.361 \mathrm{~s}$ and 5.448s respectively on TelosB which has the same design as Tmote Sky. These results are much slower compared to MicaZ's implementation because hybrid multiplication has not been implemented on TelosB/Tmote Sky in TinyECC version 0.3 . Symmetric-key encryption using Skipjack, is significantly faster than public-key encryption for the same plaintext length. Encryption takes $150 \mu \mathrm{s}$ and decryption takes $90 \mu \mathrm{s}$. Consequently, the secure discovery protocol tries to minimise the use of signature generation/verification, as well as publickey encryption/decryption by using a symmetric secret-key to convey the blinking pattern to the sensor. The key management scheme also uses Skipjack for key distribution, key renewal and key updates.

Table III shows the codesize of security components. SHA-1 uses 2,442 bytes [12] for TelosB. The wireless communication, LEDs indication and basic standard library uses $16.43 \mathrm{~Kb}$ of ROM and $0.57 \mathrm{~Kb}$ of RAM with an overall codesize of $36.20 \mathrm{~Kb}(\mathrm{ROM})$ and $3.56 \mathrm{~Kb}(\mathrm{RAM})$ for the secure discovery protocol and key management. This could be further optimised by using TinyECC version 1.0 [16] which implements Barret reduction, repeated point doubling and affine coordinate point addition and doubling. 
TABLE III

CODESIZE FOR SECURITY OPERATIONS ON TMOTE SKY

\begin{tabular}{l|c|c} 
Security Operations & ROM & RAM \\
\hline Elgamal public-key encryption & $10.42 \mathrm{~Kb}$ & $1.54 \mathrm{~Kb}$ \\
Skipjack & $4.41 \mathrm{~KB}$ & $0.45 \mathrm{~Kb}$
\end{tabular}

\section{DISCUSSION}

In contrast to wireless sensor networks for environmental monitoring where sensors relay data to sink nodes, healthcare BSNs usually rely on a patient controller that manages sensor configuration changes, i.e., monitoring thresholds, adding and removing sensors and actuators. Delegating the computational intensive security functions to the patient's controller can prolong battery life on sensors and support better security mechanisms such as signature generation and verification. However, the controller becomes a single point of failure and thus it is important that the patient protects the device from malicious tampering and theft. If stolen, the patient can manually turn off the sensors to avoid information leakage.

Human intervention is needed to replace a failed battery and restart the sensor, but results in loss of the state information regarding which patient the sensor is associated with. Consequently, we advocate that upon discovery, the sensor stores the patient's public-key in its flash and when restarted, it authenticates and re-associates with the patient device without requiring a healthcare worker's intervention. The patient's device must keep a record of the sensor's information, e.g., the shared secret-key or the sensor's public-key to facilitate re-association. The sensor can be explicitly disassociated by erasing the public-key from the flash through a management action by the healthcare worker.

The use of a shared group key has a drawback in that it does not distinguish the sender of a message from other group members. Hence, it does not guarantee non-repudiation and implies that anyone in possession of the group key can modify the message content. As a result, the group key is purely used for ensuring message confidentiality and integrity in BSNs.

Outside of a hospital environment, the controller's periodic broadcast may represent a significant loss of privacy, if it is perceived to be of a medical nature. However, adding new sensors requires intervention from a healthcare worker and is unlikely to occur outside of a clinical setting. Techniques such as those proposed in [7] could then be employed but require shared secrets (code words) to be established a priori.

\section{CONCLUSIONS AND FUTURE WORK}

We have presented an approach to securely discover sensors and associate them to the patient's controller to join the patient's BSN. The design takes account of the limitations of mobile devices/sensors - lack of screen display and constrained computational capability. Our approach to discovering sensors enables the devices to exchange security information such as public-keys and symmetric key. More importantly, we provide a practical scheme of associating sensors to the patient's controller which can only be done by an authorised healthcare worker.

We have also presented a novel key distribution and management scheme based solely on keychains, hash functions and symmetric-key encryption; it is thus very efficient and has low computational overheads. Distribution of group keys is simplified and key update messages can be authenticated easily thus facilitating efficient renewal of group keys to cater for membership changes.

Further measurements and performance analysis work are required to explore protocol trade-offs. Mitigating DoS attacks aimed at depleting resources and investigating mechanisms to detect anomalous behaviour is also required. Finally, we would like to extent our work to enable authentication between sensors and thus to be able to enforce access control policies.

\section{REFERENCES}

[1] J. Andersen and J. E. Bardram, "BLIG: A New Approach for Sensor Identification, Grouping, and Authorisation in Body Sensor Networks ," in Proc. 4th Int. Workshop on Wearable and Implantable Body Sensor Networks, Aachen, Germany, March 26 - 28, 2007.

[2] D. Liu, P. Ning, and R. Li, "Establishing Pairwise Keys in Distributed Sensor Networks," ACM Trans. on Information and System Security, vol. 8, no. 1, pp. $41-77$, Feb 2005.

[3] L. Eschenauer and V. D. Gligor, "A key-management scheme for distributed sensor networks," in Proc. 9th ACM Conf. on Computer and communications security. Washington, DC, USA: ACM, 2002.

[4] D. Balfanz, D. Smetters, P. Stewart, and H. Wong, "Talking to Strangers: Authentication in Ad-hoc Wireless Networks," in Proc. Network and Distributed System Security Symp., San Diego, 2002.

[5] F. Stajano and R. Anderson, "The Resurrecting Duckling: Security Issues for Ad-hoc Wireless Networks," in Proc. 7th Int. Workshop on Security Protocols, ser. LCNS. Springer-Verlag, 1999.

[6] C. Jiang, B. Li, and H. Xu, "An Efficient Scheme for User Authentication in Wireless Sensor Networks," in Proc. 21st Int. Conf. on Advanced Information Networking and Applications Workshops. Washington, DC, USA: IEEE Computer Society, 2007.

[7] F. Zhu, W. Zhu, M. W. Mutka, and L. M. Ni, "Expose or Not? A Progressive Exposure Approach for Service Discovery in Pervasive Computing Environments," in 3rd IEEE Int. Conf. on Pervasive Computing and Communications, 8-12 March 2005, Kauai Island, HI, USA, 2005.

[8] C. He and J. Mitchell, "Security Analysis and Improvements for IEEE 802.11i," in Proc. Network and Distributed System Security Symp., 2005.

[9] S. L. Keoh, "Security proof of secure discovery protocol for body sensor networks," Aug 2008. [Online]. Available: http://www.doc.ic.ac.uk/slk/files/bsn-proof.pdf

[10] L. Lamport, "Password Authentication with Insecure Communication," Communications of the ACM, vol. 24, no. 11, pp. 770-772, 1981.

[11] A. Perrig, R. Canetti, J. Tygar, and D. Song, "The TESLA Broadcast Authentication Protocol," RSA Cryptobytes, 2002.

[12] A. Liu, P. Kampanakis, and P. Ning, "TinyECC: Elliptic Curve Cryptography for Sensor Networks (Version 0.3)," Feb 2007. [Online]. Available: http://discovery.csc.ncsu.edu/software/TinyECC/

[13] "SEC 2: Recommended Elliptic Curve Domain Parameters," Standards for Efficient Cryptography (SEC), Certicom Research, Tech. Rep., September 2000. [Online]. Available: http://www.secg.org/collateral/sec2_final.pdf

[14] M. Rosing, Implementing Elliptic Curve Cryptography. Manning Publications Co., 1999.

[15] C. Karlof, N. Sastry, and D. Wagner, "TinySec: A Link Layer Security Architecture for Wireless Sensor Networks," in Proc. 2nd Int. Conf. on Embedded Networked Sensor Systems. Baltimore, MD, USA: ACM 2004, pp. 162-175.

[16] A. Liu and P. Ning, "TinyECC: A Configurable Library for Elliptic Curve Cryptography in Wireless Sensor Networks," North Carolina State Univ., Dept of Computer Science, Tech. Rep. TR-2007-36, Nov 2007. 